\title{
RESEARCH
}

\section{Period polynomials, derivatives of $L$-functions, and zeros of polynomials}

\author{
Nikolaos Diamantis $^{1^{*}(\mathbb{D} \text { and Larry Rolen }}{ }^{2}$
}

${ }^{*}$ Correspondence:

nikolaos.diamantis@nottingham.ac.uk

'University of Nottingham,

Nottingham, UK

Full list of author information is

available at the end of the article

\begin{abstract}
Period polynomials have long been fruitful tools for the study of values of $L$-functions in the context of major outstanding conjectures. In this paper, we survey some facets of this study from the perspective of Eichler cohomology. We discuss ways to incorporate non-cuspidal modular forms and values of derivatives of $L$-functions into the same framework. We further review investigations of the location of zeros of the period polynomial as well as of its analogue for $L$-derivatives.
\end{abstract}

Keywords: Periods of modular forms, Derivatives of $L$-functions, Eichler cohomology, Critical L-values

Mathematics Subject Classification: 11F11, 11F67

\section{Introduction}

The period polynomial provides a way of encoding critical values of $L$-functions associated with modular cusp forms that has proven very successful in the uncovering of important arithmetic properties of $L$-values. As such, its structure and properties as an object in its own right have attracted a lot of interest from various perspectives, one of the most important ones being that of Zagier, as will become apparent below. To give an idea of the uses of the period polynomial and its structure, we start by outlining its definition.

Let $f$ be an element of the space $S_{k}$ of weight $k$ cusp forms for $\mathrm{SL}_{2}(\mathbb{Z})$. The period polynomial of $f$ is the polynomial in $X$ given by

$$
r_{f}(X):=\int_{0}^{\infty} f(\tau)(\tau-X)^{k-2} \mathrm{~d} \tau .
$$

A relation with the $L$-function of $f$ is provided by the identity (cf eg. [34])

$$
r_{f}(X)=\sum_{n=0}^{k-2}\left(\begin{array}{c}
k-2 \\
n
\end{array}\right) i^{n-1} \Lambda_{f}(n+1) X^{n}
$$

where $\Lambda_{f}(s):=(2 \pi)^{-s} \Gamma(s) L_{f}(s)$ is the "completed" $L$-function of $f$.

An example of the manner by which the structure of the period polynomial leads to important arithmetic information about values of $L$-functions is Manin's Periods Theorem. The algebraic properties of $r_{f}$ (cocycle relations) combined with the arithmetic nature of $f$ (as a Hecke eigenform) lead to a certain one-dimensionality statement for $r_{f}$, which with (1.1) translates to the following proportionality relation.

(c) The Author(s) 2018. This article is distributed under the terms of the Creative Commons Attribution 4.0 International License (http://creativecommons.org/licenses/by/4.0/), which permits unrestricted use, distribution, and reproduction in any medium, provided you give appropriate credit to the original author(s) and the source, provide a link to the Creative Commons license, and indicate if changes were made. 
Theorem 1.1 Manin's Periods Theorem [27]. Let $f$ be a normalized Hecke eigenform in $S_{k}$ with rational Fourier coefficients. Then there exist $\omega_{+}(f), \omega_{-}(f) \in \mathbb{R}$ such that

$$
\Lambda_{f}(s) / \omega_{+}(f), \quad \Lambda_{f}(w) / \omega_{-}(f) \in \mathbb{Q}
$$

for all $s, w$ with $1 \leq s, w \leq k-1$ and s even, wodd.

Remark 1.2 For illustration purposes, here we gave a special case of the actual theorem which will be discussed in slightly more detail in the next section. This special case was essentially known earlier; see, for instance, $\$ 9$ of [32].

Fundamental applications such as the above theorem have motivated closer independent study of $r_{f}$, for instance as a polynomial. The aspect we will be focusing on in this survey is the location of the zeroes of $r_{f}(X)$.

The strength of techniques based on $r_{f}$ has likewise motivated the search for analogues of the period polynomial in other situations. The example we will more closely be reviewing here is an analogue of the period polynomial associated with derivatives of $L$-functions.

Derivatives of $L$-functions are the subject of some of the main current conjectures in number theory, e.g. by Birch-Swinnerton-Dyer and by Beilinson. To review a part of the latter, in an explicit formulation due to Kontsevich-Zagier [23], we recall the definition of periods, again in the form given in [23]: These are complex numbers whose real and imaginary parts have the form

$$
\int_{V} \frac{P(\mathbf{x})}{Q(\mathbf{x})} \mathrm{d} \mathbf{x}
$$

where $V$ is a domain in $\mathbb{R}^{n}$ defined by polynomial inequalities with coefficients in $\mathbb{Q}$ and $P, Q \in \mathbb{Q}\left[X_{1}, \ldots, X_{n}\right]$. The set $\mathcal{P}$ of periods contains $\pi, \log (n)(n \in \mathbb{N})$, etc. The arrangement of the following special case of Beilinson's conjecture follows [23].

Conjecture (Deligne-Beilinson-Scholl) Let $f$ be a weight $k$ Hecke eigencuspform for $S L_{2}(\mathbb{Z}), L_{f}(s)$ its L-function, and $m$ an integer. Then, if $r$ is the order of vanishing of $L_{f}(s)$ at $s=m$,

$$
L^{(r)}(m) \in \mathcal{P}[1 / \pi] .
$$

Apart from the cases $r=0$ (treated by Manin, Deligne, Beilinson, Deninger-Scholl; see [23] and the references contained there) and $r=1$ (thanks, in the case of weight 2, to Gross-Zagier [17]), this conjecture is still open. Analogues of the period polynomial for first derivatives of $L$-functions have been given in $[7,16]$. The version we will be using is

$$
\int_{0}^{\infty} f(w)(w-z)^{k-2}\left(\log (w)-\frac{\pi i}{2}\right) \mathrm{d} w .
$$

The justification for this choice will come from cohomological considerations (see Sect. 3), but, for the time being, we note that that this polynomial, in analogy with (1.1), equals

$$
-\sum_{n=0}^{k-2}\left(\begin{array}{c}
k-2 \\
n
\end{array}\right) i^{1-n} \Lambda_{f}^{\prime}(n+1) z^{k-2-n} .
$$

This polynomial has an algebraic structure that fits into the same context as that of the standard period polynomial. It was recently observed by the authors that, at least conjecturally, its zeros follow the same pattern as those of the standard period polynomial. 
Conjecture 1.3 ("Riemann hypothesis for period polynomials attached to $L$-derivatives") [11] For any Hecke eigenform of weight $k$ on $\mathrm{SL}_{2}(\mathbb{Z})$, and for each $m \in \mathbb{Z}_{\geq 0}$, the polynomial

$$
Q_{f}(z):=\sum_{n=0}^{k-2}\left(\begin{array}{c}
k-2 \\
n
\end{array}\right) i^{1-n} \Lambda_{f}^{(m)}(n+1) z^{k-2-n}
$$

has all its zeros on the unit circle. Moreover, its odd part

$$
\sum_{\substack{n=1 \\
n \text { odd }}}^{k-3}\left(\begin{array}{c}
k-2 \\
n
\end{array}\right) i^{1-n} \Lambda_{f}^{(m)}(n+1) z^{k-2-n}
$$

has all of its zeros on the unit circle, except for $0, \pm a, \pm 1 /$ a for some $a \in \mathbb{R}$.

In [11], this statement was proved in the case of Eisenstein series.

In this survey, we will review the theory of period polynomials and of the "period polynomials" attached to $L$-derivatives from a cohomological perspective. We will further survey conjectures and results about zeros of period polynomials and of their counterparts for $L$-derivatives.

\section{Period polynomials}

\subsection{Period polynomials of cusp forms}

Set $\Gamma:=\mathrm{PSL}_{2}(\mathbb{Z})$. This group is generated by $S=\left(\begin{array}{cc}0 & -1 \\ 1 & 0\end{array}\right)$ and $T=\left(\begin{array}{ll}1 & 1 \\ 0 & 1\end{array}\right)$ (or, more precisely, by their images under the natural projection of $\mathrm{SL}_{2}(\mathbb{Z})$ onto $\left.\Gamma\right)$. The only relations are

$$
S^{2}=(S T)^{3}=1
$$

For $\tau \in \mathfrak{H}$, let $f(\tau)=\sum_{n=1}^{\infty} a_{n} e^{2 \pi i n}$ be a cusp form of even weight $k$ for $\Gamma$. A way to define the period polynomial associated with $f$ is as a polynomial in $z$ of degree $\leq k-2$ given by

$$
r_{f}(z):=\int_{0}^{\infty} f(\tau)(\tau-z)^{k-2} \mathrm{~d} \tau
$$

The origin of this definition goes back (at least) to Poincaré (cf. [30]) in the context of work on Abelian integrals (a fact brought to our attention by [12]). Since then, the period polynomial has been interpreted in several ways, each providing new insight and leading to important applications.

We will review two that are most relevant for our purposes.

\section{a. Eichler cohomology}

Firstly, Eichler [13] and Shimura [32] viewed them as periods of iterated integrals that are now called Eichler integrals:

$$
F(z)=(k-2) ! \int_{\infty}^{z} \int_{\infty}^{z_{1}} \ldots \int_{\infty}^{z_{k-2}} f\left(z_{k-1}\right) \mathrm{d} z_{k-1} \ldots \mathrm{d} z_{1}=\int_{\infty}^{z} f(\tau)(\tau-z)^{k-2} \mathrm{~d} \tau .
$$

The relation of $F$ with $r_{f}$ is given by

$$
F(-1 / z) z^{k-2}-F(z)=r_{f}(z)
$$

This identity can be viewed as the starting point of an algebraic approach to the study of the period polynomial which has far-reaching implications. It first implies that $r_{f}$ induces 
a 1-cocycle in Eichler cohomology, which we will now define. Since we will later need cocycles in more general cases, we recall the general definition of cocycles.

Let $M$ be a right $\Gamma$-module. For $i \geq 0$, we call $i$-cochain for $\Gamma$ with coefficients in $M$ a map from $\Gamma^{i}$ to $M$. The group they form is denoted by $C^{i}(\Gamma, n)$. The differential $d^{i}: C^{i}(\Gamma, M) \rightarrow C^{i+1}(\Gamma, M)$ is given by

$$
\begin{aligned}
& \left(d^{i} \sigma\right)\left(g_{1}, \ldots, g_{i+1}\right) \\
& \quad:=\sigma\left(g_{2}, \ldots, g_{i+1}\right) \cdot g_{1}+\sum_{j=1}^{i}(-1)^{j} \sigma\left(g_{1}, \ldots, g_{j+1} g_{j}, \ldots, g_{i+1}\right)+(-1)^{i+1} \sigma\left(g_{1}, \ldots, g_{i}\right) .
\end{aligned}
$$

Set $Z^{i}(\Gamma, M)=\operatorname{ker}\left(d^{i}\right)$ for the group of $i$-cocyles and, when $i \geq 1, B^{i}(\Gamma, M)=$ $d^{i-1}\left(C^{i-1}(\Gamma, M)\right)$ for the group of $i$-coboundaries. We set $B^{0}:=0$. The group $H^{i}(\Gamma, M):=$ $Z^{i}(\Gamma, M) / B^{i}(\Gamma, M)$ is the set of $i$-cohomology classes. For instance, a 1-cocycle $\phi$ is a map from $\Gamma$ to $M$ such that

$$
\phi\left(g_{2} g_{1}\right)=\phi\left(g_{2}\right) \cdot g_{1}+\phi\left(g_{1}\right) \quad \text { for all } g_{1}, g_{2} \in \Gamma \text {. }
$$

Both $Z^{i}$ and $H^{i}$ are endowed with a Hecke action which we will not define but mention because it plays an important role in an application below. Detailed expositions can be found in $[6,32]$.

In Eichler cohomology, we apply this construction with $M$ the space $P_{k-2}$ of polynomials of degree $\leq k-2$. The action $\left.\right|_{2-k}$ of $\Gamma$ on $P_{k-2}$ or, more generally, on functions on $\mathfrak{H}$ is:

$$
\left(\left.P\right|_{2-k} \gamma\right)(z):=P(\gamma z) j(\gamma, z)^{k-2}, \quad z \in \mathfrak{H}, \gamma \in \Gamma,
$$

where $j\left(\left(\begin{array}{ll}* & * \\ c & d\end{array}\right), \tau\right):=c \tau+d$. With this notation, (2.4) is rewritten as

$$
\left.F\right|_{2-k} S-F=r_{f}
$$

We now consider the map $\sigma_{f}: \Gamma \rightarrow P_{k-2}$ defined by first setting $\sigma_{f}(S)=r_{f}$ and $\sigma_{f}(T)=0$ and extending to $\Gamma$ according to (2.6). This, in view of (2.1), gives a well-defined map because, with (2.7),

$$
\sigma_{f}\left(S^{2}\right)=\left.\sigma_{f}(S)\right|_{2-k} S+\sigma_{f}(S)=\left.r_{f}\right|_{2-k} S+r_{f}=\left.F\right|_{2-k}\left(S^{2}-S\right)+\left.F\right|_{2-k}(S-1)=0,
$$

and likewise $\sigma_{f}\left((S T)^{3}\right)=0$.

Note that (2.7) does not mean that $r_{f}$ is a 1-coboundary in $B^{1}\left(\Gamma, P_{k-2}\right)$. It would only mean that if $F$ were in $P_{k-2}$. It is a coboundary in a larger space but, as we just saw, this fact suffices to show that $r_{f}$ is 1-cocycle in $Z^{1}\left(\Gamma, P_{k-2}\right)$. This technique is used often in constructions of cocycles and will reappear in the sequel.

It is possible to express the value of $\sigma_{f}$ at every $\gamma \in \Gamma$ by a simple formula:

$$
\sigma_{f}(\gamma)(z)=\int_{\gamma^{-1} \infty}^{\infty} f(\tau)(\tau-z)^{k-2} \mathrm{~d} z
$$


A fundamental fact is that Eichler cohomology parametrizes modular forms by means of the Eichler-Shimura isomorphism. For general $f$ in the space $M_{k}$ of all weight $k$ modular forms for $\mathrm{SL}_{2}(\mathbb{Z})$, we define $\sigma_{f}$ by

$$
\sigma_{f}(\gamma)(z):=\int_{\gamma^{-1} \tau_{0}}^{\tau_{0}} f(\tau)(\tau-z)^{k-2} \mathrm{~d} z
$$

where $\tau_{0} \in \mathfrak{H}$ is fixed. If $\bar{g}$ is the function obtained by conjugating the values of $g$, we define $r_{\bar{f}}$ by a similar formula involving integration of antiholomorphic differentials. Then, a version of the Eichler-Shimura isomorphism can be stated as

Theorem 2.1 (Eichler-Shimura isomorphism) Let $\sigma$ be the map assigning to $(f, \bar{g}) \in$ $M_{k} \oplus \overline{S_{k}}$ the 1-cocycle $\sigma_{f}+\sigma_{\bar{g}}$ and let $\pi$ be the natural projection of $Z^{1}\left(\Gamma, P_{k-2}\right)$ onto $H^{1}\left(\Gamma, P_{k-2}\right)$. Then $\pi \circ \sigma$ is a Hecke-equivariant isomorphism.

From this viewpoint, the period polynomial of a cusp form $f$ can be redefined as the value at the involution $S$ of the image of $f$ under the Eichler-Shimura map $\sigma$.

\section{b. Critical values of $L$-functions}

A second interpretation of the period polynomial is as a generating function of critical values of $L$-functions. As usual, we define the $L$-function of a modular form $f(z)=$ $\sum_{n=0}^{\infty} a_{n} e^{2 \pi i n z}$ by

$$
L_{f}(s):=\sum_{n=1}^{\infty} \frac{a_{n}}{n^{s}} \quad(\text { for } \operatorname{Re}(s) \gg 0),
$$

and the completed $L$-function by

$$
\Lambda_{f}(s):=(2 \pi)^{-s} \Gamma(s) L_{f}(s) .
$$

The function $\Lambda_{f}$ has a meromorphic continuation to the entire complex plane with possible (simple) poles at 0 and $k$, and it satisfies the functional equation (see, e.g. [19], Chapt. 7):

$$
\Lambda_{f}(s)=i^{k} \Lambda_{f}(k-s)
$$

It further has an integral expression:

$$
\Lambda_{f}(s)=\int_{1}^{\infty}(f(i v)-a(0)) v^{s-1} \mathrm{~d} v+i^{k} \int_{1}^{\infty}(f(i v)-a(0)) v^{k-s-1} \mathrm{~d} v-\frac{a(0)}{s}-\frac{a(0) i^{k}}{k-s}
$$

When $f$ is a cusp form, $\Lambda_{f}(s)$ is entire and (2.11) becomes the classical Mellin transform:

$$
\Lambda_{f}(s)=\int_{0}^{\infty} f(i v) v^{s-1} \mathrm{~d} v .
$$

The values of the $L$-functions of Hecke eigencuspforms are of fundamental importance, among other reasons, because they are, at least conjecturally, closely connected with arithmetic and classical arithmetic questions. For example, a part of the Birch-SwinnertonDyer conjecture implies that, if $L_{f}(1) \neq 0$, for a weight 2 cusp form of a certain type, then a 
specific polynomial Diophantine equation has at most finitely many solutions. This is part of the order 0 Birch-Swinnerton-Dyer conjecture and has been proven in $[4,17,24,25]$.

Among the values of $L$-functions, the values at the integers within the critical strip $0<\operatorname{Re}(s)<k$ are called critical and were the first ones to be studied. Using the binomial theorem and (2.12), one can show that the period polynomial naturally encodes the critical $L$-values:

$$
r_{f}(z)=-i \sum_{j=0}^{k-2}\left(\begin{array}{c}
k-2 \\
j
\end{array}\right)(i z)^{j} \Lambda_{f}(j+1) .
$$

Remark 2.2 The cohomological properties of $r_{f}$ discussed in Part a. can be translated to analogous statements here. Notably, if we rewrite the equations proving that $\sigma_{f}$ is welldefined (e.g. (2.8)) in terms of (2.13), we are led to Manin's important "Eichler-Shimura relations" (Prop. 2.1 of [27]). A crucial implication of these relations, discussed in the next application, is that $Z^{1}\left(\Gamma, P_{k-2}\right)$ (more precisely, the part of this space corresponding to the odd part of the polynomials in $P_{k-2}$ ) is defined by a linear system of equations with rational coefficients.

Application of $a$. and $b$. We will combine the above two interpretations of $r_{f}$ to illustrate the power of the period polynomial with the following result.

Theorem 2.3 (Manin's Periods Theorem [27]) Letf be a normalized Hecke eigencuspform in $S_{k}$ and let $K_{f}$ be the field obtained by adjoining to $\mathbb{Q}$ the Fourier coefficients off. There exist $\omega_{+}(f), \omega_{-}(f) \in \mathbb{R}$ such that

$$
\Lambda_{f}(s) / \omega_{+}(f), \quad \Lambda_{f}(w) / \omega_{-}(f) \in K_{f}
$$

for all $s, w$ with $1 \leq s, w \leq k-1$ and $s$ even, $w$ odd.

Besides Manin's proof in [27], other proofs stressing different aspects include those of Shokurov ([31], geometric methods on Kuga-Sato varieties), Zagier ([35], using RankinSelberg method and Rankin-Cohen brackets), Shimura ([33], by another variant of the Rankin-Selberg method), the first author and O'Sullivan ([10], by a variation of a method of [22] which uses holomorphic projection and Cohen's kernel), etc.

Sketch of Proof of 2.3 The Hecke eigencuspform $f$ generates a one-dimensional eigenspace of $S_{k}$. By Theorem 2.1, this is mapped isomorphically to a one-dimensional eigenspace of $H^{1}\left(\Gamma, P_{k-2}\right)$ and, in fact, the restriction of that map to just the even (resp. odd) powers of the polynomial induces an isomorphism too. It can also be proved that this map sends $f$ to a one-dimensional eigenspace $A_{f}$ of the even (resp. odd) part of $Z^{1}\left(\Gamma, P_{k-2}\right)$ not just of $H^{1}\left(\Gamma, P_{k-2}\right)$. By Remark 2.2, $Z^{1}\left(\Gamma, P_{k-2}\right)$ is defined over $\mathbb{Q}$ and thus $A_{f}$ is defined over $K_{f}$. Since $\operatorname{dim}\left(A_{f}\right)=1$, this implies that there is a $c \in \mathbb{C}$ such that the even (resp. odd) part of $\sigma_{f}(S)=r_{f}$ equals $c P^{+}$for an even polynomial $P^{+} \in K[z]$ (resp. $c P^{-}$for an odd polynomial $\left.P^{-} \in K[z]\right)$. With (2.13), this implies that quotients of critical $L$-values of the same parity belong to $K_{f}$.

\subsection{Period polynomials of non-cuspidal modular forms}

The definition (2.2) no longer applies in the case that $f$ is not cuspidal because the integral may fail to converge at the end points. However, it is possible to modify the definitions so 
that they include general modular forms as well. To our knowledge, the first one to give a general definition and systematically study it was Zagier in [34] (Grosswald [18], starting from a different departure point, also worked with a similar object and proved a explicit expression for it.) In the case of general $f \in M_{k}$ the "first definition" (2.2) was replaced by

$$
\begin{aligned}
\widetilde{r}_{f}(z)= & \int_{i}^{\infty}\left(f(\tau)-a_{0}\right)(\tau-z)^{k-2} \mathrm{~d} \tau+\int_{0}^{i}\left(f(\tau)-a_{0} \tau^{-k}\right)(\tau-z)^{k-2} \mathrm{~d} \tau \\
& +\frac{a_{0}}{k-1}\left((z-i)^{k-1}+\frac{(1+i z)^{k-1}}{z}\right) \\
= & \left.\left(\int_{\infty}^{i}\left(f(\tau)-a_{0}\right)(\tau-z)^{k-2} \mathrm{~d} \tau+\frac{a_{0}}{k-1}(i-z)^{k-1}\right)\right|_{2-k}(S-1) .
\end{aligned}
$$

The "second definition" (2.13) was replaced by

$$
\tilde{r}_{f}(z)=-i \sum_{j=0}^{k-2}\left(\begin{array}{c}
k-2 \\
j
\end{array}\right)(i z)^{j} \Lambda_{f}(j+1)+\frac{a_{0}}{k-1}\left(z^{k-1}+z^{-1}\right)
$$

This extended definition is then used in [34] to state and prove an expression of a striking generating function involving period polynomials over a basis of $M_{k}$ as a quotient of products of values of the classical Jacobi theta function.

A difference from the case of cusp forms is that $\widetilde{r}_{f}$ is not in $P_{k-2}$ when $f$ is not cuspidal. Recently, it was shown in [3] that it is possible to define the period polynomial of Eisenstein series so that it stays within $P_{k-2}$. Set

$$
r_{f}(z)=\left.\left(\int_{\infty}^{z}\left(f(w)-a_{0}\right)(w-z)^{k-2} \mathrm{~d} w+a_{0} \int_{0}^{z}(w-z)^{k-2} \mathrm{~d} w\right)\right|_{2-k}(S-1) .
$$

This definition was made in a vastly general context by Brown in [3] which included general iterated Shimura integrals and originated from an integral at a tangential base point at infinity. In this more general setting, it is proved that $r_{f} \in P_{k-2}$ and that it induces a 1-cocycle. Brown's extension of the period polynomial was also motivated by important applications. For example, he used it to express non-critical values in terms of multiple modular values.

A way to compare this definition with that of [34] is to consider the explicit form of the Eichler cocycle it induces. Set

$$
v_{f}(z):=\int_{\infty}^{z}\left(f(w)-a_{0}\right)(w-z)^{k-2} \mathrm{~d} w+\frac{a_{0}}{k-1} z^{k-1} .
$$

Then, with the definition of $d^{0}$ in (2.5) we define $\sigma_{f}:=d^{0} v_{f}$.

It is clear that $r_{f}=\sigma_{f}(S)$. On the other hand, the associated cocycle of $\widetilde{r}_{f}$ is given by $\widetilde{\sigma}_{f}:=d^{0} \widetilde{v}_{f}$, where

$$
\widetilde{v}_{f}(z):=\int_{\infty}^{i}\left(f(w)-a_{0}\right)(w-z)^{k-2} \mathrm{~d} w+\frac{a_{0}}{k-1}(i-z)^{k-1}
$$

and where $d^{0}$ is defined by the same formula as (2.5) but its domain is enlarged to $C^{0}(\Gamma, \mathbb{C}(z))$. 
As mentioned above, in [3] it is proven in more general form that $\sigma_{f}$ takes values in $P_{k-2}$. This can also be seen by the identity shown in [11] [eq. (8)]:

$$
\begin{aligned}
\sigma_{f}(\gamma)= & \int_{\gamma^{-1} i}^{i} f(w)(w-z)^{k-2} \mathrm{~d} w \\
& +\left.\left(\int_{\infty}^{i}\left(f(w)-a_{0}\right)(w-z)^{k-2} \mathrm{~d} w+a_{0} \int_{0}^{i}(w-z)^{k-2} \mathrm{~d} w\right)\right|_{2-k}(\gamma-1) .
\end{aligned}
$$

From this, it is also clear that $\sigma_{f}$ is "canonical" in the sense that it belongs to the same cohomology class as the image of $f$ under the Eichler-Shimura isomorphism (Theorem 2.1). With this definition of the cocycle $r_{f}$, we then have

$$
r_{f}(z)=-i \sum_{j=0}^{k-2}\left(\begin{array}{c}
k-2 \\
j
\end{array}\right)(i z)^{j} \Lambda_{f}(j+1)
$$

(see the proof of Lemma 7.1 in [3]). Consider the case when $f=E_{k}$, the Eisenstein series

$$
E_{k}(\tau)=-\frac{B_{k}}{2 k}+\sum_{n=1}^{\infty} \sigma_{k-1}(n) e^{2 \pi i n \tau}
$$

where $B_{a}$ is the $a$ th Bernoulli number. Then Lemma 7.1 of [3] shows that the above equation takes the form

$$
r_{E_{k}}(z)=-\frac{(k-2) !}{2} \sum_{j=0}^{k / 2-2} \frac{B_{2 j+2}}{(2 j+2) !} \frac{B_{k-2 j-2}}{(k-2 j-2) !} z^{2 j+1}+\frac{(k-2) !}{2} \frac{\zeta(k-1)}{(2 \pi i)^{k-1}}\left(1-z^{k-2}\right)
$$

and, by Proposition of pg. 453 of [34] [or (2.14)], we have

$$
\tilde{r}_{E_{k}}(z)=-\frac{(k-2) !}{2} \sum_{j=-1}^{k / 2-1} \frac{B_{2 j+2}}{(2 j+2) !} \frac{B_{k-2 j-2}}{(k-2 j-2) !} z^{2 j+1}+\frac{(k-2) !}{2} \frac{\zeta(k-1)}{(2 \pi i)^{k-1}}\left(1-z^{k-2}\right) .
$$

\subsection{Zeros of period polynomials}

Having argued the case for the conceptual importance of period polynomial and for its usefulness due to its structure, it becomes clear that it is of interest to examine it for its own sake, as a polynomial. We review work on its zeros as a polynomial.

For $k \in 2 \mathbb{N}$, Murty et al. [28] studied the Ramanujan polynomial

$$
\sum_{j=0}^{k / 2} \frac{B_{2 j}}{(2 j) !} \frac{B_{k-2 j}}{(k-2 j) !} z^{2 j}
$$

and proved the following result.

Theorem 2.4 [28] All non-real zeros of the Ramanujan polynomial are on the unit circle. 
With (2.19), the Ramanujan polynomial equals the odd part of $-2 z \widetilde{r}_{E_{k}}(z) /(k-2)$ !

Because of (2.8), the circle is a natural "line of symmetry" for the period polynomials, and therefore results such this can be thought of as a "Riemann Hypothesis" for period polynomials. This viewpoint was adopted in [29] where similar statements are connected to Manin's theory of "zeta polynomials" $Z_{f}(s)$. These are versions of the period polynomials that send the unit circle to $\operatorname{Re}(s)=\frac{1}{2}$ and satisfy the functional equation $Z_{f}(1-s)= \pm Z_{f}(s)$.

In [26], Lalín and Smyth studied the zeroes of the "Ramanujan polynomials"

$$
\mathcal{R}_{k}(z):=\sum_{j=0}^{k / 2} \frac{B_{2 j}}{(2 j) !} \frac{B_{k-2 j}}{(k-2 j) !} z^{2 j}+\frac{\zeta(k-1)}{(2 \pi i)^{k-1}}\left(z^{k-1}-z\right) .
$$

Theorem 2.5 [26] For each $k \in 2 \mathbb{N}$, the zeroes of $\mathcal{R}_{k}$ all lie on the unit circle.

From a modular perspective, our interest in these polynomials is that, by (2.19) they are the full $-2 z \widetilde{r}_{E_{k}}(z) /(k-2)$ !. In the sequel, we shall be concerned with analogues of Theorem 2.4 for derivatives of Eisenstein $L$-series. In particular, it will serve as the main motivation for our first steps towards understanding our broader conjectures for entire modular forms spaces.

An interesting recent interpretation of the period polynomial of Eisenstein series and of their zeros in view of Ramanujan's "formula" for $\zeta(2 m+1)$ is discussed in [2]. In the same paper, a question is raised about a variation of the Ramanujan polynomial:

$$
p_{m}(z)=\frac{\zeta(2 m+1)}{2}\left(1-z^{2 m}\right)-\frac{(2 \pi i)^{2 m+1}}{2} \sum_{n=1}^{m} \frac{B_{2 n}}{(2 n) !} \frac{B_{2 m-2 n+2}}{(2 m-2 n+2) !} z^{2 n-1}
$$

Note that $p_{k / 2-1}(z)=(2 \pi i)^{k-1} r_{E_{k}}(z) /(k-2)$ !. In Remark 7.4 of [2], it is asked whether $p_{m}$ and $p_{m}^{-}(z) / z$ (where $p_{m}^{-}$is the odd part of $p_{m}$ ) are unimodular. In [11], we proved the second part of this conjecture.

Analogous results have been proved for cusp forms. For example, Conrey, Farmer, and Imamoglu [5] have proved that, apart from five "trivial" real zeroes, all zeroes of the odd part of the period polynomial of a cusp form lie on the unit circle.

Theorem 2.6 (Conrey, Farmer, and Imamoḡlu) If $f$ is a cuspidal Hecke eigenform on $\mathrm{SL}_{2}(\mathbb{Z})$, then the odd part of $r_{f}$ has zeroes at $0, \pm \frac{1}{2}, \pm 2$. The remainder of the zeros lies on the unit circle.

A similar picture exists for the full-period polynomials $r_{f}$ for Hecke eigencuspforms $f$. However, in this case, there are no trivial zeros, and all zeros of $r_{f}$ lie on the unit circle. This is summarized in the following result, shown by El-Guindy and Raji in [14] for level 1 and for general level $N$ by Jin et al. in [20].

Theorem 2.7 (El-Guindy and Raji and Jin, Ma, Ono, and Soundararajan) Iff is a Hecke eigencuspforms on $\Gamma_{0}(N)$ for any $N$, then all zeroes of the period polynomial $r_{f}$ lie on the unit circle.

Remark 2.8 Explicit approximations for the exact locations of the zeroes were given in [20]. 
The proofs of the above results are based on the origin of the period polynomial as a cocycle. In particular, the behaviour of $r_{f}$ under the action of the involution $S$ imposes a special structure on the polynomial (it is a self-inversive polynomial). This allows for a more convenient investigation of the location of the zeros thanks to the following result.

Lemma 2.9 Theorem 2.2 of [14]) If $h(z)$ is a polynomial of degree $n$ with all zeros inside the unit disk $|z| \leq 1$, then for any $d \geq n$ and $\lambda$ on the unit circle, the polynomial

$$
z^{d-n} h(z)+\lambda z^{n} \bar{h}(1 / z)
$$

has all its zeroes on the unit circle, provided that it is not identically zero.

Statements of this type have a long history which can be traced back to Hermite (see the Addendum of [26] for an account) but, in this form, the proposition has been proved in [14].

In this way, the problem of locating the zeros of $r_{f}$ is reduced to locating the zeros of the polynomial $h$ associated with $r_{f}$ through Lemma 2.9. This is achieved by bounds and monotonicity statements for values of $L$-functions appearing in the coefficients of $h$. The results cited above are proved by using different such bounds and monotonicity statements.

\section{3 "Period polynomial" for derivatives of $L$-functions}

Beilinson's conjecture, part of which is stated in Conj. 1, pertains to values of derivatives of L-functions and, as mentioned in the introduction, very little is known about the case of order greater than 0 . This has motivated many approaches to the study of values of derivatives. We will outline one, due to Goldfeld and the first author (see $[6,7,16]$ and the later works by them and their collaborators: $[1,8,9]$ ) that incorporates these values into the Eichler cohomology setting. In the cited papers, only the cuspidal case was studied but here we will describe the general case as that was described in [11].

\subsection{First derivatives}

We first recall the Dedekind eta function

$$
\eta(\tau):=e^{\frac{2 \pi i \tau}{24}} \prod_{n=0}^{\infty}\left(1-e^{2 \pi i n \tau}\right)
$$

and then set $u(\tau):=2 \log (\eta(\tau))$. For each $\gamma \in \Gamma$, this function satisfies

$$
u(\gamma \tau)=u(\tau)+\log (j(\gamma, \tau))+c_{\gamma}
$$

for some $c_{\gamma} \in \mathbb{C}$. In particular, $c_{S}=-\frac{\pi i}{2}$.

Let, as before, $f$ be a modular form of weight $k$ for $\mathrm{SL}_{2}(\mathbb{Z})$. With the definition (2.5), we set $\sigma_{f}:=d^{1} v_{f}$ where

$$
\begin{aligned}
v_{f}(\gamma):= & \int_{\infty}^{z}\left(f(w)-a_{0}\right)(w-z)^{k-2}(u(\gamma w)-u(w)) \mathrm{d} w \\
& +a_{0} \int_{i}^{z}(w-z)^{k-2}(u(\gamma w)-u(w)) \mathrm{d} w
\end{aligned}
$$


It can be proved that, although $v_{f}$ is a cochain that takes values in the space $\mathcal{O}$ of holomorphic functions on the upper-half plane, $\sigma_{f}$ takes values in the much smaller space of polynomials of degree $\leq k-2$. Since, further, it is in the image of the differential map $d^{1}$, we deduce:

Lemma 3.1 (Lemma 3.3 of [11]) The map $\sigma_{f}$ is a 2-cocycle in $P_{k-2}$.

As mentioned above, this construction extends the corresponding one or cusp forms given in [7]. This is the content of the following proposition which, further, expresses $\sigma_{f}$ in a way which makes the analogy with the standard polynomial (2.9) more transparent.

Proposition 3.2 Letf be a cusp form of weight $k$ for $\Gamma$. Then

$$
\begin{aligned}
\sigma_{f}\left(\gamma_{1}, \gamma_{2}\right) & =\int_{\gamma_{1}^{-1} \infty}^{\infty} f(w)(w-z)^{k-2}\left(u\left(\gamma_{2} w\right)-u(w)\right) d w \\
& =\left.\int_{\infty}^{\gamma_{1} \infty} f(w)(w-z)^{k-2}\left(u\left(\gamma_{2} w\right)-u(w)\right) d w\right|_{2-k} \gamma_{1} .
\end{aligned}
$$

The connection with values of derivatives of $L$-functions is given by

Proposition 3.3 (Prop. 3.5 of [11]) Set

$$
P(z)=\sum_{n=0}^{k-2}\left(\begin{array}{c}
k-2 \\
n
\end{array}\right) \frac{i^{1-n}}{(n+1)^{2}} z^{k-2-n} .
$$

Then

$$
\sigma_{f}(S, S)=-\sum_{n=0}^{k-2}\left(\begin{array}{c}
k-2 \\
n
\end{array}\right) i^{1-n} \Lambda_{f}^{\prime}(n+1) z^{k-2-n}+a(0)\left(\left.P\right|_{2-k}(1+S)\right)(z) .
$$

The proposition is stated in general, but, for cuspidal $f$, the analogy to (2.17) is obvious.

\subsection{Zeros of "period polynomials" for $L$-derivatives}

In light of the analogy with the standard period polynomial, it is natural to ask whether similar patterns in the distribution of zeros occur in "period polynomials" for derivatives of $L$-functions. Inspired by the behaviour exhibited by ordinary period polynomials as described in Theorems 2.6 and 2.7, the authors searched for similar properties for polynomials built from $L$-derivatives in [11]. There, the analogous period polynomials were defined to be polynomials

$$
Q_{f}(z):=\sum_{n=0}^{k-2}\left(\begin{array}{c}
k-2 \\
n
\end{array}\right) i^{1-n} \Lambda_{f}^{\prime}(n+1) z^{k-2-n},
$$

in direct analogy with (1.1) and the following conjecture was formulated.

Conjecture 3.4 For any Hecke eigenform of weight $k$ on $\mathrm{SL}_{2}(\mathbb{Z})$ the polynomial $Q_{f}(z)$ has all its zeros on the unit circle. Moreover, the odd part of $Q_{f}(z)$ has all of its zeros on the unit circle, except for trivial zeros at 0 and $\pm a, \pm 1 /$ a for some real number $a$.

The evidence for this conjecture was both theoretical and experimental. The former was provided by our proof of the second part of Conjecture 3.4 in the case of Eisenstein series. This is, at the same time, the analogue of the main result of [28] on period polynomials of Eisenstein series. 
Theorem 3.5 [11] If $4 \mid k$, all nonzero zeroes of the odd part of $Q_{E_{k}}$ lie on the unit circle.

As in the case of the standard period polynomial, the pivot of the proof is the cohomological origin of the "period polynomial" $Q_{E_{k}}$ which allows us to study it as a self-inversive polynomial. On the other hand, we were then able to use more general theorems about locations of zeros (Eneström-Kakeya Theorem $[15,21])$. This is because our construction parallels Brown's version of the period polynomial of Eisenstein series $\left(r_{E_{k}}\right)$ and not that of [34] $\left(\tilde{r}_{E_{k}}\right)$ which, with its two extra terms, takes us away from the coefficient module of polynomials.

At first glance, since in Theorem 2.4 there are further real roots (in addition to 0), the conclusion of Theorem 3.5 appears to not be analogous with its counterpart Theorem 2.4. The reason for this is that, whereas the subject of Theorem 2.4 is Zagier's version of the period polynomial of Eisenstein series, the subject of Theorem 3.5 is a polynomial which extends Brown's version of the period polynomial of Eisenstein series. The analogue of Theorem 2.4 for Brown's version of the period polynomial of Eisenstein series was stated as a question in [2] and has the same conclusion as Theorem 3.5 (as shown in [11]).

Question Are all nonzero zeroes of the odd part of

$$
p_{k / 2-1}(z)=\frac{(2 \pi i)^{k-1}}{(k-2) !} r_{E_{k}}(z)
$$

on the unit circle?

The experimental evidence for the truth of Conjecture 3.4 is also very convincing and will be outlined along the respective discussion of higher derivatives.

We end this section by noting that it would be very interesting to interpret the role of the number $a$ in the statement of the conjecture, and in particular to find an explanation for them as "trivial zeros", as was the case for the zeros with $a=2$ in Theorem 2.6.

\subsection{The case of higher derivatives}

An advantage of the approach on derivatives of $L$-function discussed here is that it includes in a natural way higher derivatives about which, as mentioned earlier, very little is known. Therefore, any progress by this method in the case of first derivatives might lead to insights for higher derivatives as well.

The cohomological tool enabling to extend the constructions of Sect. 3.1 to higher derivatives is cup products. This, in the case we need it, is defined as a map

$$
\cup: C^{1}(\Gamma, \mathcal{O}) \otimes C^{m}(\Gamma, \mathcal{O}) \rightarrow C^{m+1}(\Gamma, \mathcal{O})
$$

given by

$$
\left(\phi_{1} \cup \phi_{2}\right)\left(\gamma_{1}, \gamma_{2}, \ldots, \gamma_{m+1}\right):=\phi_{1}\left(\gamma_{1}\right)\left(\left.\phi\left(\gamma_{2}, \ldots, \gamma_{m+1}\right)\right|_{0} \gamma_{1}\right) .
$$

A crucial property that that cup products of cocycles are cocycles. For $\phi_{i} \in C^{1}(\Gamma, \mathcal{O})$, we set:

$$
\phi_{1} \cup \cdots \cup \phi_{n}:=\phi_{1} \cup\left(\phi_{2} \cup\left(\cdots\left(\phi_{n-1} \cup \phi_{n}\right) \cdots\right)\right) \in C^{n}(\Gamma, \mathcal{O}) .
$$

If $v$ is the 1-cocycle given by $\left.\gamma \rightarrow u\right|_{0}(\gamma-1)$ (with $u$ as in Sect. 3.1), we set, for $n \in \mathbb{N}$,

$$
V_{n}:=v \cup v \cup \cdots \cup v \quad(n \text { times). }
$$

As mentioned above, this will be a $n$-cocycle. 
Let $v_{f} \in C^{n}(\Gamma, \mathcal{O})$ be given by

$$
\begin{aligned}
v_{f}\left(\gamma_{1}, \ldots, \gamma_{n}\right)= & \int_{\infty}^{z}\left(f(w)-a_{0}\right)(w-z)^{k-2} V_{n}\left(\gamma_{1}, \ldots, \gamma_{n}\right)(w) \mathrm{d} w \\
& +a_{0} \int_{i}^{z}(w-z)^{k-2} V_{n}\left(\gamma_{1}, \ldots, \gamma_{n}\right)(w) \mathrm{d} w
\end{aligned}
$$

Setting $\sigma_{f}:=d^{n} v_{f}$, we arrive at the following analogue of Lemma 3.1 for higher cocycles.

Lemma 3.6 (Lemma 3.6 of [11]) The map $\sigma_{f}$ takes values in $P_{k-2}$ and thus gives an $(n+1)$-cocycle in $P_{k-2}$.

Finally, the analogue of Proposition 3.3 is

Proposition 3.7 (Prop. 3.7 of [11]) For each $m \in \mathbb{N}$, set

$$
P(z)=\sum_{n=0}^{k-2}\left(\begin{array}{c}
k-2 \\
n
\end{array}\right) \frac{i^{1-n}}{(-n-1)^{m+1}} z^{k-2-n} .
$$

Then

$$
\begin{aligned}
(-1)^{m} \sigma_{f}(S, \ldots S)= & \sum_{n=0}^{k-2}\left(\begin{array}{c}
k-2 \\
n
\end{array}\right) i^{1-n} \Lambda_{f}^{(m)}(n+1) z^{k-2-n} \\
& -a(0) m !\left(\left.P\right|_{2-k}\left(1+(-1)^{m+1} S\right)\right)(z),
\end{aligned}
$$

where $\sigma_{f}$ has $m+1$ arguments.

This proposition led us to formulate Conjecture 1.3 as the general version of Conjecture 3.4.

Conjecture 3.8 For any Hecke eigenform of weight $k$ on $\mathrm{SL}_{2}(\mathbb{Z})$, and for each $m \in \mathbb{Z}_{\geq 0}$, the polynomial

$$
Q_{f}(z):=\sum_{n=0}^{k-2}\left(\begin{array}{c}
k-2 \\
n
\end{array}\right) i^{1-n} \Lambda_{f}^{(m)}(n+1) z^{k-2-n}
$$

has all its zeros on the unit circle. Moreover, the odd part

$$
\sum_{\substack{n=1 \\
n \text { odd }}}^{k-3}\left(\begin{array}{c}
k-2 \\
n
\end{array}\right) i^{1-n} \Lambda_{f}^{(m)}(n+1) z^{k-2-n}
$$

has all of its zeros on the unit circle, except for zeros at 0 and $\pm a, \pm 1 /$ for some $a \in \mathbb{R}$.

We were able to prove the Eisenstein series case of the second part of this conjecture too, but we had to truncate the "lower order" terms from the $\Lambda_{f}^{(m)}$ appearing in $Q_{f}$. The precise construction is slightly complicated but the essence of the theorem is entirely analogous to Theorem 3.5 (see Theorem 4.2 of [11]).

The experimental evidence for Conjecture 3.8 in the case of both the first and the higher derivatives was based on computer search. In particular, the authors used SAGE to check that the norms of all zeroes of all full-period polynomials with $m \leq 3$ and $k \leq 50$ were 
within $10^{-10}$ of 1 . The structure of the second part of the conjecture was made on the basis of similar computational experiments.

\section{Author details}

${ }^{1}$ University of Nottingham, Nottingham, UK, ${ }^{2}$ Hamilton Mathematics Institute \& Trinity College Dublin, Dublin, Ireland.

\section{Dedication}

Dedicated to Don Zagier in honor of his 65th birthday.

\section{Publisher's Note}

Springer Nature remains neutral with regard to jurisdictional claims in published maps and institutional affiliations.

Received: 31 August 2017 Accepted: 21 December 2017 Published online: 6 February 2018

\section{References}

1. Bruggeman, R., Choie, Y., Diamantis, N.: Holomorphic automorphic forms and cohomology. Memoirs of the AMS arXiv:1404.6718 (in press)

2. Berndt, B., Straub, A.: Ramanujan's formula for $\zeta(2 n+1)$, chapter 2 of the book. In: Montgomery, H., Nikeghbali, A., Rassias, M. (eds.) Exploring the Riemann Zeta Function, pp. 13-34. Springer, Berlin (2017)

3. Brown, F.: Multiple modular values and the relative completion of the fundamental group of $M_{1,1}$. arXiv:1407.5167 (under review)

4. Coates, J., Wiles, A.: On the conjecture of Birch and Swinnerton-Dyer. Invent. Math. 39, 223-251 (1977)

5. Conrey, J.B., Farmer, D.W., Imamoglu, Ö.: The nontrivial zeros of period polynomials of modular forms lie on the unit circle. Int. Math. Res. Not. 20, 4758-4771 (2013)

6. Diamantis, N.: Special values of higher derivatives of L-functions. Forum Math. 11(1), $229-252$ (1999)

7. Diamantis, N.: Hecke operators and derivatives of L-functions. Compos. Math. 125(1), 39-54 (2001)

8. Diamantis, N.: The geometry of certain cocycles associated to derivatives of $L$-functions. Forum Math. 17(5), 739-752 (2005)

9. Diamantis, N., Neururer, M., Strömberg, F.: A correspondence of modular forms and applications to values of $L$-series. Res. Number Theory 1, 27 (2015)

10. Diamantis, N., O'Sullivan, C.: Kernels of L-functions of cusp forms. Math. Ann. 346(4), 897-929 (2010)

11. Diamantis, N., Rolen, L.: Eichler cohomology and zeros of polynomials associated to derivatives of $L$-functions. arXiv:1704.02667 (under review)

12. Duke, W., Imamoglu, Ö., Tóth, Á.: Rational period functions and cycle integrals. Abhandlungen aus dem Mathematischen Seminar der Universität Hamburg 80(2), 255-264 (2010)

13. Eichler, M.: Eine Verallgemeinerung der Abelsche Integrale. Math. Z. 67, 267-298 (1957) (under review)

14. El-Guindy, A., Raji, W.: Unimodularity of zeros of period polynomials of Hecke eigenforms. Bull. Lond. Math. Soc. 46(3), 528-536 (2014)

15. Eneström, G.: Ramarque sur un théorème relatif aux recines de l'equation $a_{n} x_{n}+\cdots+a_{0}=0$ où tous les coefficients sont réels et positifs, Tôhoku Math. J. 18, 34-36 (1920). Translation of a Swedish article in Ofversigt of Konogl. Vertenskaps Akademiens Förhandlingar 50, 405-415 (1893)

16. Goldfeld, D.: Special values of derivatives of L-functions. Number theory (Halifax, NS, 1994), 159-173, CMS Conference Proceedings, vol. 15. American Mathematical Society, Providence, RI (1995)

17. Gross, B., Zagier, D.: Heegner points and derivative of L-series. Invent. Math. 85, 225-320 (1986)

18. Grosswald, E.: Die Werte der Riemannschen Zeta-funktion an ungeraden Argumentstellen, Nachr. Akad. Wiss. Göttingen, 9-13 (1970)

19. Iwaniec, H.: Topics in Classical Automorphic Forms. Graduate Studies in Mathematics, vol. 17. AMS, Providence (1991)

20. Jin, S., Ma, W., Ono, K., Soundararajan, K.: The Riemann Hypothesis for period polynomials of modular forms. Proc. Natl. Acad. Sci. USA 113(10), 2603-2608 (2016)

21. Kakeya, S:: On the limits of the roots of an algebraic equation with positive coefficients. Tohoku Math. J. 2, 140-142 (1912-1913)

22. Kohnen, W., Zagier, D.: In: Rankin, R.A. (ed.) Modular Forms with Rational Periods in Modular Forms. Ellis Horwood, Chichester, pp. 197-249 (1984)

23. Kontsevich, M., Zagier, D.: Periods, Mathematics Unlimited -2001 and Beyond, pp. 771-808. Springer, Berlin (2001)

24. Kolyvagin, V.: Finiteness of $E(\mathbb{Q})$ and III $(E, \mathbb{Q})$ for a subclass of Weil curves. Izv. Akad. Nauk SSSR Ser. Mat. 52(3), 522-540 (1988)

25. Kolyvagin, V:: The Mordell-Weil and Shafarevich-Tate groups for Weil elliptic curves. IzV. Akad. Nauk SSSR Ser. Mat. 52(6), 1154-1180 (1988)

26. Lalín, M., Smyth, C.: Unimodularity of zeros of self-inversive polynomials. Acta Math. Hungar. 138(1-2), 85-101 (2013). Addendum, Acta Math. Hungar. 147 (2015), no. 1, 255-257

27. Manin, Y.T.: Periods of cusp forms and p-adic Hecke series. Math. Sb., pp. 371-393 (1973)

28. Murty, M., Smyth, C., Wang, R.: Zeros of Ramanujan polynomials. J. Ramanujan Math. Soc. 26, $107-125$ (2011)

29. Ono, K., Rolen, L., Sprung, F.: Zeta-polynomials for modular form periods. Adv. Math. 306, 328-343 (2017)

30. Poincaré, H.: Sur les invariants arithmètiques. J. für Reine u. Ang. Math. 129, 89-150 (1905)

31. Shokurov, V.: Shimura integrals of cusp forms. Izv. Akad. Nauk SSSR Ser. Mat. 44(3), 670-718 720 (1980)

32. Shimura, G.: Sur les intégrales attachées aux formes automorphesm. J. Math. Soc. Jpn. 11, 291-311 (1959) 
33. Shimura, G.: The special values of the zeta functions associated with cusp forms. Commun. Pure Appl. Math. 29(6), 783-804 (1976)

34. Zagier, D.: Periods of modular forms and Jacobi theta functions. Invent. Math. 104(3), 449-465 (1991)

35. Zagier, D.: Modular forms whose Fourier coefficients involve zeta-functions of quadratic fields. Modular functions of one variable, VI (Proceedings of Second International Conference, University of Bonn, Bonn, 1976) LNM 627. Springer, Berlin, pp. 105-169 (1977) 